Artigos especiais

Esta seção destina-se à publicação de artigos de autores convidados.

Os textos serão publicados no idioma original

\title{
La Declaración Universal sobre Bioética y Derechos Humanos - ampliación democrática para una sociedad mas justa
}

\section{Universal Declaration on Bioethics and Human Rights - a democratic amplification for a more fair society}

\author{
Alya Saada \\ Organização das Nações Unidas para a Educação, a Ciências e a Cultura \\ (UNESCO), México DF, México. \\ a.saada@unesco.org
}

Resumen: Este artículo enfoca algunos de los aspectos de la Declaración Universal sobre Bioética y Derechos Humanos que pueden provocar más impactos en términos de políticas públicas para la inclusión social y superación de la pobreza. Apunta la importancia de la incorporación de eses principios en el tratamiento de los problemas bioéticos que enfrentan las sociedades contemporáneas. Discute aún aspectos controvertidos de la dicha Declaración bajo la perspectiva delineada por los demás tratados de Derechos Humanos.

Palabras-clave: Declaración sobre Bioética. Derechos Humanos. Desigualdad. Pobreza.

\begin{abstract}
This paper focuses some aspects of Universal Declaration on Bioethics and Human Rights, which may have more impacts in terms of public policies with the aim of social inclusion and overcoming of poverty. It shows the importance of incorporating these principles to the treatment of bioethical problems faced by actual societies. The text also discusses some controversial aspects of the mentioned Declaration under the perspective outlined by the other treaties of Human Rights.
\end{abstract}

Key words: Declaration on Bioethics. Human Rights. Inequality. Poverty. 


\section{Revista Brasileira de Bioética}

La Constitución de la Organización de las Naciones Unidas para la Educación, la Ciencia y la Cultura (UNESCO), establecida en 1945, declara en su preámbulo que:

"La grande y terrible guerra que acaba de terminar no hubiera sido posible sin la negación de los principios democráticos de la dignidad, la igualdad y el respeto mutuo de los hombres... que una paz fundada exclusivamente en acuerdos políticos y económicos entre gobiernos no podría obtener el apoyo unánime, sincero y perdurable de los pueblos y que, por consiguiente, esa paz debe basarse en la solidaridad intelectual y moral de la humanidad" (1).

Como definido en el Artículo 1 de su Constitución, el propósito de la UNESCO es:

"...contribuir a la paz y a la seguridad estrechando, mediante la educación, la ciencia y la cultura, la colaboración entre naciones, a fin de asegurar el respeto universal a la justicia, a la ley, a los derechos humanos y a las libertades fundamentales (...) sin distinción de raza, sexo, idioma o religión" (1).

La ética y el respeto de los derechos humanos se encuentran, así, en el acta fundacional de la Organización. A lo largo de los 62 años de su existencia, UNESCO fue promoviendo a través de la educación, de la ciencia y de la cultura, elaborando las herramientas para el desarrollo del diálogo, de la comprensión mutua y de la colaboración entre los pueblos, así como entre los científicos y la comunidad académica mundial condición necesaria desde su punto de vista para el establecimiento de la solidaridad intelectual y moral de la humanidad y para asegurar las bases de la paz.

Con el crecimiento y la diversificación de los saberes científicos y tecnológicos durante la segunda mitad del Siglo XX e inicio del Siglo XXI, se desarrollaron nuevos conocimientos y habilidades tecnocientíficas, lo que resultó en el surgimiento de otras preocupaciones y cuestionamientos de orden ético, relacionados con los adelantos de la ciencia y su aplicación en el campo de la vida humana. Surgió 
la necesidad de desarrollar principios universales basados en valores éticos comunes, que orientasen los avances científicos y el desarrollo tecnológico, así como las transformaciones sociales derivadas, con el fin de determinar los desafíos que surgen en el ámbito de la ciencia y la tecnología y establecer guiás éticas para el desarrollo científico.

Es en este marco que, en 1993, se estableció el Programa de la Ética de la Ciencia y de la Tecnología en la UNESCO, con el propósito de reflexionar sobre los problemas éticos fundamentales suscitados por los adelantos científicos y tecnológicos y su aplicación en las ciencias de la vida, a fin de definir un marco ético universal común en materia de bioética. La acción de la UNESCO en este campo se desarrolló según tres ejes principales correspondiendo a las funciones centrales de la Organización: normativa, educativa e informativa.

La primera, aplicada al campo de la ciencia, se traduce en la elaboración de recomendaciones y de instrumentos normativos y legales dirigidos a los Estados Miembros, que permiten establecer leyes y reglas correspondientes a los problemas éticos inéditos provocados por el desarrollo de la ciencia y la tecnología aplicadas a los seres vivos, particularmente los seres humanos. Para cumplir con este objetivo, fueron establecidos dos órganos internacionales:

1) El Comité Internacional de Bioética (CIB), compuesto por 36 expertos pertenecientes a las diferentes regiones del mundo y elegidos a título personal. La función del CIB es identificar y debatir sobre los problemas éticos emergentes y elaborar propuestas de instrumentos normativos que, posteriormente, se someterán a los representantes de los órganos dirigentes de la UNESCO;

2) El Comité Intergubernamental de Bioética (CIGB), compuesto por representantes de los Gobiernos de los Estados miembros de la Organización, cuya función es evaluar, revisar y adoptar las propuestas del CIB.

Se debe subrayar que el CIB y el CIGB son espacios de reflexión y de discusión de carácter interdisciplinario y plural que reflejan la diversidad cultural y la política internacional.

Desde entonces, se promulgaron tres Declaraciones: 


\section{Revista Brasileira de Bioética}

1) La Declaración Universal del Genoma Humano y los Derechos Humanos, adoptada por la Conferencia General de la UNESCO en 1997 y también por unanimidad, en 1998, por la Asamblea General de las Naciones Unidas (2);

2) En 2003, la Declaración Internacional sobre los Datos Genéticos Humanos (3);

3) En 2005 la Declaración Universal sobre Bioética y Derechos Humanos (4).

Las dos primeras declaraciones reflejan las preocupaciones y problemas éticos así como los debates internacionales de los años 1990. Están enfocadas hacia los aspectos bio-médicos y tecnológicos así como a las implicaciones sociales, económicas y legales que supondría el uso del conocimiento de la secuencia completa del genoma humano. Tratan de asuntos controvertidos como la reproducción artificialmente asistida; el inicio y fin de la vida; los transplantes de tejidos y de órganos; la clonación; los derechos y la dignidad de la persona humana como sujeto de experimentación científica; el consentimiento informado; la privacidad y confidencialidad de los datos genéticos; la beneficencia y no maleficencia. En fin, tratan de reglamentar los diversos aspectos que tienen relación con el manejo del genoma humano, su protección contra posibles abusos y de las intervenciones técnicas sobre la vida humana desde la concepción hasta la muerte.

La Declaración Universal sobre Bioética y Derechos Humanos (4), adoptada por la Conferencia General de la UNESCO el 19 de Octubre de 2005, representa un cambio paradigmático del concepto la bioética. Esta es la primera oportunidad que se enuncia un marco comprehensivo universal de los principios de este campo de estudios, con una definición amplia de la bioética que incluye la responsabilidad social de los gobiernos en términos de salud y de bienestar colectivo, la preservación del medio ambiente y de la diversidad cultural, además de la lucha contra la pobreza y la exclusión social en todas sus formas.

Así, la reflexión ética y bioética deja de ser un asunto solamente para expertos, de científicos, de investigadores y de "sabios". Es asunto de todas y cada una de las personas. Los adelantos técnico- 
científicos que caracterizan nuestro mundo tienen un impacto sobre la conducta humana, individual y colectiva, sobre las relaciones interpersonales, los valores morales y éticos que rigen y regulan la vida social. El conjunto de los artículos de la Declaración busca abarcar el rol de las condiciones que producen tal impacto, visando responder de manera objetiva a las demandas por soluciones éticas para los problemas provenientes de esas transformaciones.

Como lo anuncia el Artículo I de la Declaración, las cuestiones éticas relacionadas con la medicina, las ciencias de la vida y sus aplicaciones tecnológicas sobre los seres humanos, se deben examinar teniendo en cuenta no sólo el respeto debido a la dignidad humana, sino también el respeto universal y la observancia de los derechos y las libertades fundamentales (4). Bajo tal perspectiva, las dimensiones sociales, culturales, legales y ambientales conforman los elementos indisociables de la vida humana.

La revolución biológica, genética y tecnológica, ocurrida durante los 50 últimos años, tuvo como resultado el desarrollo de un poder casi sin límites sobre la vida en general y la vida humana en particular. Por lo tanto, este desarrollo aparece como desconectado de las finalidades originalmente concebidas para el conocimiento científico, que es la realización de una vida mejor para la humanidad.

Hoy ya disponemos de todos los conocimientos y saberes científicos y tecnológicos para: acabar con la mayor parte de las enfermedades infecciosas a nivel mundial; procurar alimentación y hogar con agua limpia para todas las personas; proporcionar educación y herramientas económicas y culturales objetivando permitir a cada persona, cualquier sea su origen o pertenencia, desarrollar sus capacidades humana y vivir según los criterios de una vida humana digna. Sin embargo, la brecha entre los pobres y los ricos sigue ampliándose con consecuencias inadmisibles para una gran parte de la humanidad, que carece de condiciones mínimas para una vida sana.

La pobreza, el analfabetismo, la enfermedad y la exclusión son los atributos de la vida de miles de millones de seres humanos. Actualmente, en el mundo, alrededor de 1.200 millones de personas subsisten con menos de 1 dólar por día, 800 millones sufren de desnutrición y 153 millones de niños menores de cinco años tienen peso insuficiente. De hecho, sólo el 20\% más rico de la sociedad acapara el 80\% de los 


\section{Revista Brasileira de Bioética}

recursos humanos del mundo, mientras que el 20\% más pobre, obtiene sólo el 0.5\% (5).

El ingreso total de los 500 indivíduos más ricos del mundo es superior al ingreso de los 416 millones más pobres. Mas allá de estos extremos, los 2.500 millones de personas que viven con menos de dós dólares al día - y que representan el 40\% de la población mundial obtienen sólo el 5\% del ingresso mundial. El 10\% más rico, casi todo ello formado por habitantes de los países de ingressos altos, consigue el 54\% (6).

Esta división desigual de los recursos mundiales se refleja directamente en condiciones de vida marcadas por la vulnerabilidad: existen 781 millones de personas analfabetas; 76,8 millones de niños y niñas sin acceso a la escuela (7). Según el mismo Informe Mundial del Programa de las Naciones Unidas para el Desarrollo - PNUD - de 2006, más de 1.000 millones de personas se ven privadas del derecho a un agua limpia y 2.600 millones no tienen acceso a un saneamiento adecuado (8). El agua y la falta de saneamiento son relacionados con $1,8 \%$ de las muertes infantiles anuales, lo que eclipsa el número de muertes asociada a los conflictos violentos (9).

Frente a tales números urge tomar decisiones políticas basadas en valores éticos tanto a nivel global, como regional y local, para que el progreso esté orientado al desarrollo humano teniendo en cuenta los derechos de las generaciones futuras, como viene subrayado en el Artículo 16 de la Declaración (4).

Además, la Declaración apunta la necesidad de articular el progreso en el mejoramiento de las condiciones sociales, incluyendo la salud, el acceso a alimentación sana y agua saludable, así como a la educación sin discriminación ninguna (4). Debe darse la prioridad a los grupos vulnerables, que en realidad forman la gran parte de la población mundial. La pobreza, la exclusión y el analfabetismo son violaciones de los derechos humanos y como tales deben ser erradicados en un mundo que ya dispone de la riqueza, de los conocimientos y de las técnicas necesarias para este fin.

El Artículo 14 titulado Responsabilidad social y salud centra en esos aspectos y hace un llamado a los gobiernos para cumplir con sus responsabilidades políticas en términos de salud pública, proporcionando a sus ciudadanos: a) una atención médica de calidad y a los 
medicamentos esenciales, especialmente para la salud de las mujeres y los niños; b) el acceso a alimentación y agua adecuadas; c) la supresión de la marginación y exclusión de personas por cualquier motivo; d) la reducción de la pobreza y el analfabetismo (4).

Se debe subrayar que el Artículo 14 fue introducido tardíamente en el texto final, propuesto por los representantes de países de América Latina, Asia y África. En este ámbito se debe mencionar el papel de liderazgo cumplido por los miembros de la Red Latino-Americana y del Caribe de Bioética de la UNESCO (REDBIOETICA), quienes participaron de modo decisivo en la redacción de la Declaración y en la movilización de los gobiernos de la Región. El artículo fue adoptado después de un largo y tenso debate público en la Asamblea General del CIGB, dada la diversidad de posiciones políticas relacionadas con la problemática, y simboliza un avance muy significativo en materia de ética y derechos humanos.

Es importante también mencionar el Artículo 12 titulado Respeto a la Diversidad Cultural y el Pluralismo, que representa una innovación crítica y controvertida. A ese respeto es interesante ver el análisis en el artículo de Pierre Sané en esta misma edición. Los críticos (o detractores) de la Declaración consideran que este principio puede ser invocado para justificar atentados y violaciones a los derechos humanos y a las libertades fundamentales. De hecho, tenemos muchos ejemplos de violaciones en diversos países, en nombre de la tradición, de creencias y de valores culturales. Tales violaciones son la razón por la cual se precisa que el principio de la diversidad cultural "no habrá de invocarse para atentar a la dignidad humana y las libertades fundamentales" (4). Así, está explicitado en el artículo que los derechos a la diversidad cultural y el pluralismo no se sobreponen (y no pueden se sobreponer) a los derechos y libertades fundamentales.

Al garantizar la dignidad humana y las libertades fundamentales, la Declaración reconoce, implícitamente, que los individuos son las bases de toda la diversidad cultural y pluralidad en cualquiera sociedad. Hay que recordar que el respecto de la diversidad cultural es un logro universal de la comunidad internacional y se encuentra explicitado en la Declaración Universal sobre la Diversidad Cultural de la UNESCO (5).

Esa Declaración, aprobada en el 2 de Noviembre 2001 por unani- 


\section{Revista Brasileira de Bioética}

midad por los Estados Miembros de la Organización, reafirma la convicción de estos países que el diálogo intercultural es el mejor garante de la paz, elevando la diversidad cultural a la categoría de "patrimonio común de la humanidad", "tan necesaria para el género humano como la diversidad biológica para los organismos vivos" (5).

En cuanto al Artículo 17, titulado Protección del Medio Ambiente, la Biosfera y la Biodiversidad, se debe leer teniendo en mente el Convenio de las Naciones Unidas sobre la Diversidad Biológica, del 5 de junio de 1992, que afirma en su Artículo 1:

"Los objetivos del presente convenio, son la conservación de la diversidad biológica, la utilización sostenible de sus componentes y la participación justa y equitativa en los beneficios que se deriven de la utilización de los recursos genéticos, mediante, entre otras cosas, un acceso adecuado a esos recursos y una transferencia apropiada de las tecnologías pertinentes, teniendo en cuenta todos los derechos sobre esos recursos y a esas tecnologías, así como una financiación apropiada" (6).

Es preciso subrayar aún la interdependencia y la complementariedad de los diversos instrumentos normativos internacionales existentes, que conforman avances indiscutibles en términos de derecho internacional. Esos instrumentos responden a la complejidad de nuestro mundo cuyos componentes biológicos, sociales, culturales, económicos y ambientales están conectados a nivel global.

La Declaración Universal sobre Bioética y Derechos Humanos abre nuevos espacios para que la reflexión ética y proporciona herramientas necesarias para los gobiernos puedan establecer leyes, normas y legislaciones adecuadas en el campo bioético. Se necesitan acciones por parte de los legisladores, de los responsables de la salud, médicos, bioeticistas, abogados, investigadores y docentes, en todos los campos, para concretar los principios adquiridos en la realidad cotidiana de los pueblos.

En América Latina y Caribe, el papel cumplido por la UNESCO, desde 2002, fue enfocado a la creación y el desarrollo de una Red de Bioética - la REDBIOETICA - cuyos objetivos principales son: la reflexión sobre los problemas bioéticos específicos en los países de la 
región; la investigación y la publicación de textos; el intercambio de información; la organización de encuentros científicos; y el desarrollo de la educación en el campo de la bioética.

Unos de los resultados más importante realizado por la REDBIOETICA, mencionado más arriba, fue la introducción de la dimensión social en el texto de la Declaración Universal sobre Bioética y Derechos Humanos, enfocando aspectos como: justicia; equidad; responsabilidad social de los gobiernos; lucha en contra de la pobreza, exclusión social y analfabetismo; acceso a alimentación adecuada, los cuales quedaron reflejados en el Artículo 14. Para la UNESCO este resultado deriva de una ampliación democrática y refleja la realidad del mundo actual, una realidad que tenemos de cambiar.

\section{Referencias}

1. UNESCO. Constitución de la Organización de las Naciones Unidas para la Educación, la Ciencia y la Cultura. Em: http://unesdoc.unesco.org/images/ 0012/001255/125590s.pdf\#page=7. Constitución de la Organización de las Naciones Unidas para la Educación, la Ciencia y la Cultura. Acesso em: 19/12/2006.

2. UNESCO. Declaración Universal del Genoma Humano y los Derechos Humanos. Em: http://www.upo.es/general/investigar/otri/otri_docu/pn/ UNESCOgenoma.pdf Declaración Universal sobre el Genoma y Derechos Humanos. Acesso em: 19/12/2006.

3. UNESCO. Declaración Internacional sobre los Datos Genéticos Humanos. Em: http://unesdoc.unesco.org/images/0014/001461/146180S.pdf Declaración Universal sobre Bioética y Derechos Humanos. Acesso em: 19/12/2006. 4. UNESCO. Declaración Universal sobre Bioética y Derechos Humanos. Paris, Conferencia General de la UNESCO 19 de Octubre de 2005. Em: www. bioetica.catedraunesco.unb.br . Acesso em: 19/12/2006.

5. ONU. ¿̇Por qué son importante los objetivos del Milenio? Programa de las Naciones Unidas para el Desarrollo (PNUD). Folleto Informativo sobre la Aplicación de la Declaracion del Milenio de las Naciones Unidas, 2003.

6. PNUD. Informe sobre el Desarrollo Humano, 2005. pp. 4-5.

7. UNESCO. Informe de Seguimiento sobre la Educación para Todos en el Mundo, 2006. p.2.

8. PNUD. "Prefácio". In: Op.cit. p.V.

9. PNUD. Op.cit. p.3

10. UNESCO.Declaración Universal de la UNESCO sobre la Diversidad Cultural. http://www.cdi.gob.mx/transparencia/declara_diversidad.pdf Declaración Universal de la UNESCO sobre la Diversidad Cultural. Acesso em: 


\section{Revista Brasileira de Bioética}

19/12/2006.

11. ONU. Convenio de las Naciones Unidas sobre la Diversidad Biológica. Rio de Janeiro, Conferencia General de la Organización de las Naciones Unidas, 5 de Junio de 1992. Em: http://www.biodiv.org/doc/legal/cbd-es.pdf Convenio sobre la Diversidad Biologica. Acesso em: 19/12/2006.

Recebido: 8/11/2006 Aprovado: 4/12/2006 